\title{
Multi-Physics Simulations of Molten Chloride Fast Reactor using Nek5000 and PROTEUS-NODAL
}

Nuclear Science and Engineering Division 


\begin{abstract}
About Argonne National Laboratory
Argonne is a U.S. Department of Energy laboratory managed by UChicago Argonne, LLC under contract DE-AC02-06CH11357. The Laboratory's main facility is outside Chicago, at 9700 South Cass Avenue, Argonne, Illinois 60439. For information about Argonne and its pioneering science and technology programs, see www.anl.gov.
\end{abstract}

\title{
DOCUMENT AVAILABILITY
}

Online Access: U.S. Department of Energy (DOE) reports produced after 1991 and a growing number of pre-1991 documents are available free at OSTI.GOV (http://www.osti.gov/), a service of the US Dept. of Energy's Office of Scientific and Technical Information.

Reports not in digital format may be purchased by the public from the National Technical Information Service (NTIS):

U.S. Department of Commerce

National Technical Information Service

5301 Shawnee Rd

Alexandra, VA 22312

www.ntis.gov

Phone: (800) 553-NTIS (6847) or (703) 605-6000

Fax: (703) 605-6900

Email: orders@ntis.gov

Reports not in digital format are available to DOE and DOE contractors from the Office of Scientific and Technical Information (OSTI):

U.S. Department of Energy

Office of Scientific and Technical Information

P.O. Box 62

Oak Ridge, TN 37831-0062

www.osti.gov

Phone: (865) 576-8401

Fax: (865) 576-5728

This report was prepared as an account of work sponsored by an agency of the United States Government. Neither the United States Government nor any agency thereof, nor UChicago Argonne, LLC, nor any of their employees or officers, makes any warranty, exp ress or implied, or assumes any legal liability or responsibility for the accuracy, completeness, or usefulness of any information, apparatus, product, or process disclosed, or represents that its use would not infringe privately owned rights. Reference herein to any specific commercial product, process, or service by trade name, trademark, manufacturer, or otherwise, does not necessarily constitute or imply its endorsement, recommendation, or favoring by the United States Government or any agency thereof. The views and opinions of document authors expressed herein do not necessarily state or reflect those of the United States Government or any agency the reof, Argonne National Laboratory, or UChicago Argonne, LLC.
} 


\section{Multi-Physics Simulations of Molten Chloride Fast Reactor using Nek5000 and PROTEUS-NODAL}

prepared by

Y.S. Jung, J. Fang, D. Shaver, and B. Feng

Nuclear Science and Engineering Division, Argonne National Laboratory

July 30,2020 



\section{ABSTRACT}

This report documents the FY20 work to demonstrate a specific multi-physics capability within NEAMS tools to model molten chloride fast reactors (MCFR). Specifically, the CFD code Nek5000 and the neutronics code PROTEUS-NODAL were coupled off-line to calculate the delayed neutron precursor distribution within the flowing fuel salts in an MCFR core.

An MCFR benchmark problem and a 3D test problem based on a conceptual MSR design were successfully solved through the coupled simulation of Nek5000 and PROTEUS-NODAL. In the coupled simulations for these test problems, Nek5000 provided high-quality flow field information, and PROTEUS-NODAL performed the neutron diffusion calculation along with the precursor drift model. The impact of precursor drift on the core status was also investigated. Additionally, the precursor drift model implemented in PROTEUS-NODAL was verified by making use of the reference precursor distribution generated from Nek5000. Results of these test problems suggest that the multi-physics capability of Nek5000 and PROTEUS-NODAL was properly developed for tracking delayed neutron precursor drift. 


\section{Table of Contents}

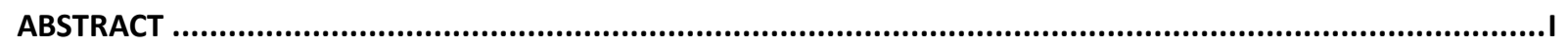

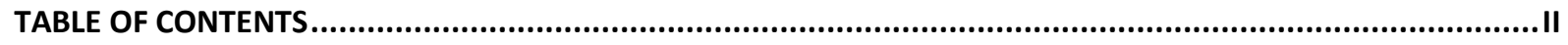

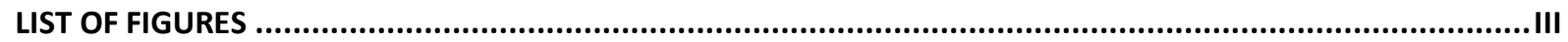

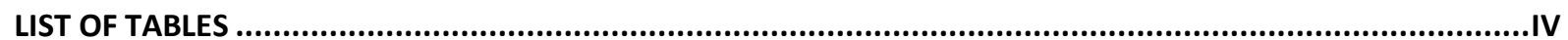

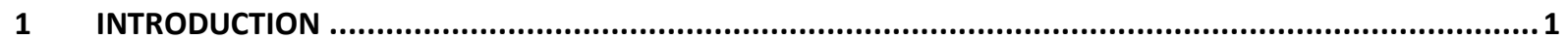

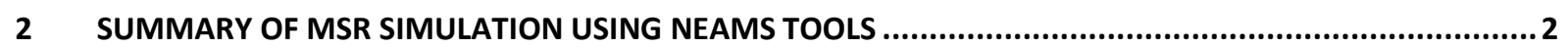

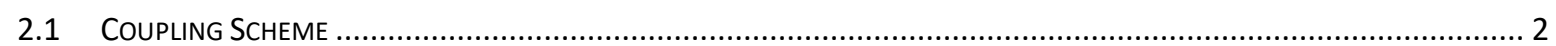

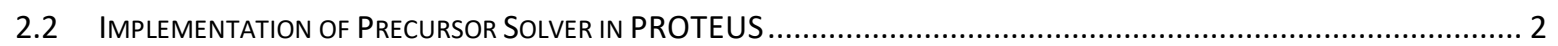

3 MCFR BENCHMARK PROBLEM .......................................................................................

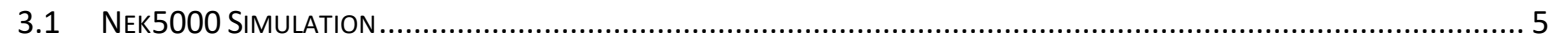

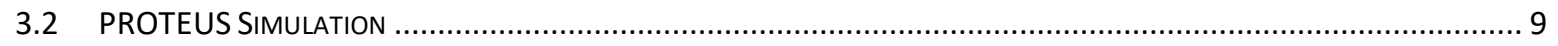

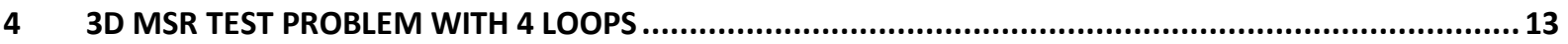

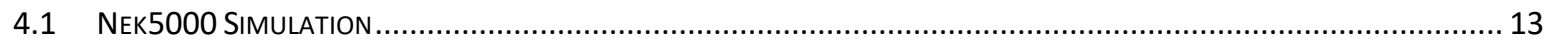

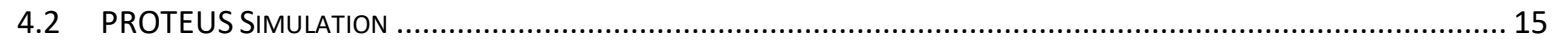

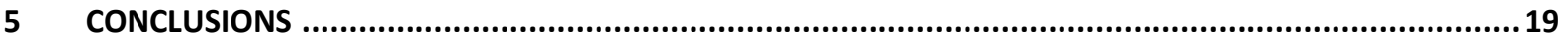

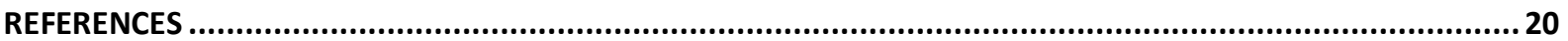




\section{LIST OF FIGURES}

Figure 2-1. Calculation flow of Nek5000 and PROTEUS-NODAL coupled simulation [1].... 2 Figure 2-2. Two-level mesh structure for PROTEUS-NODAL with precursor drift ............... 4

Figure 3-1. Computational mesh for 2D core flow case.................................................. 5

Figure 3-2. Momentum source generates a pressure jump in the outer channel which drives

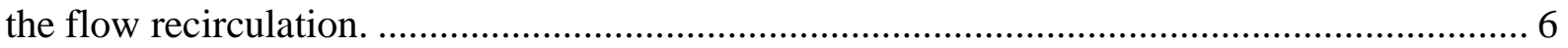

Figure 3-3. Velocity and temperature profiles in the laminar flow scenario. ......................... 6

Figure 3-4. Precursor generation rates of different families inside the $2 \mathrm{D}$ core...................... 7

Figure 3-5. Steady-state precursor concentrations for different precursor families.................. 8

Figure 3-6. PROTEUS model for MCFR benchmark problem. ..................................... 10

Figure 3-7. Precursor distribution of family 1 for MCFR benchmark problem. .................... 11

Figure 3-8. Precursor distribution of family 3 for MCFR benchmark problem. ................... 11

Figure 3-9. Comparisons of precursor distributions obtained using Nek5000 and PROTEUS-

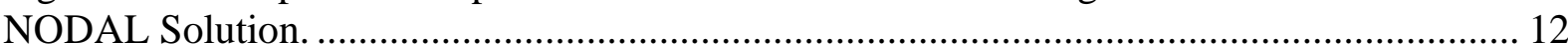

Figure 4-1. Computational mesh for a 3D MSR core with inlet (bottom) and outlet (top) pipes.

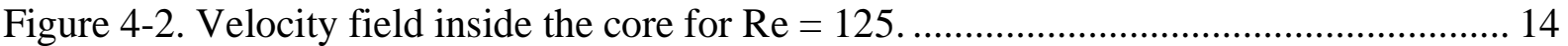

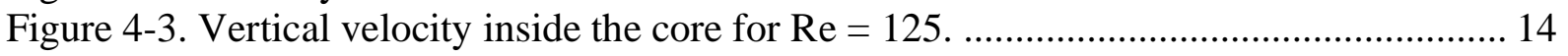

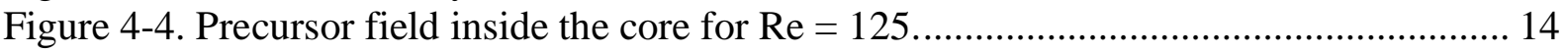

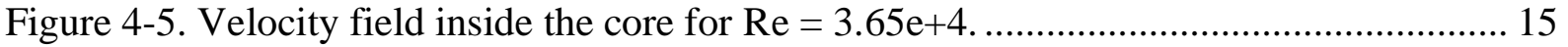

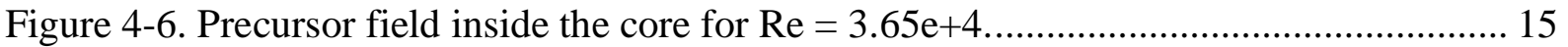

Figure 4-7. Modeling of PROTEUS-NODAL for 3D MSR test problem with 4 loops. ......... 16

Figure 4-8. Velocity and precursor distributions used in precursor drift calculations for 3D

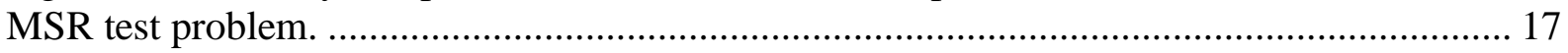

Figure 4-9. Precursor distribution of 3D MSR test problem (circulation period of $10 \mathrm{sec}$ ).... 17

Figure 4-10. Changes of precursor distributions of 3D MSR test problem with Different

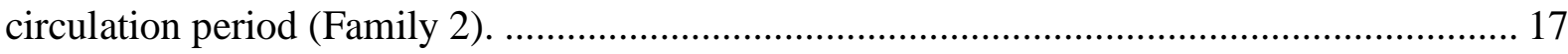




\section{LIST OF TABLES}

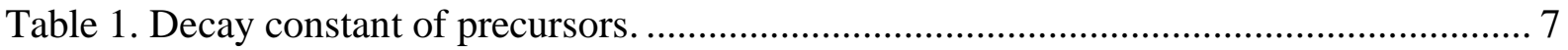

Table 2. Material properties of fuel salt and reflector at the reference temperature................. 8

Table 3. Simulation parameters corresponding to the prototypical core flow conditions. ........ 9

Table 4. Fuel Properties of MCFR benchmark problem.................................................. 9

Table 5. Flow conditions for MCFR benchmark problem.............................................. 10

Table 6. k-eff for MCFR benchmark problem................................................................ 10

Table 7. Reactivity parameters of MCFR benchmark problem with stationary fuel condition.

Table 8. $\beta$-eff of MCFR benchmark problem wits flowing fuel conditions. ........................... 12

Table 9. Eigenvalue results of 3D MSR test problem.................................................... 18 


\section{Introduction}

The advanced multi-physics simulation capabilities have been actively developed at Argonne National Laboratory by combining the NEAMS tools. As increasing interests of molten salt reactor (MSR) concept in industry, the multi-physics capability targeting on MSR application was developed under the Reactor Product Line (RPL) in the DOE-NEAMS program. [1] In this effort made in FY2019, the computational fluid dynamics (CFD) code Nek5000 [2] and the neutronics code PROTEUS-NODAL [3] were coupled to accomplish the detailed modeling and simulation of re-distribution of delayed neutron precursor caused by flowing fuels, commonly referred to the precursor drift. And the proof-of-principle tests were only performed using the developed multiphysics tool. This work is focused on demonstrating the multi-physics simulation capability of NEAMS tools for the modeling and simulation of MSR application.

As effort to demonstrate the capabilities, the MCFR benchmark and the 3D MSR test problems were solved through the coupled simulation of Nek5000 and PROTEUS-NODAL. The MCFR benchmark problem was prepared based on a challenging problem set proposed by industry. The computational domain was described with Cartesian lattice structure, which explicitly includes the active core and external pipe as well. Then, the Nek5000 and PROTEUS-NODAL calculations were performed, and the precursor distributions were obtained with the various flow conditions ranging from laminar to turbulent cases. The impact of precursor drift on the reactivity parameters were investigated. The 3D MSR test problem was prepared based on the conceptual MSR design that has the cylindrical active core region and the 4-loop inlet/outlet pipes attached to top and bottom of radial periphery. The velocity field formed in the active core region was examined using the Nek5000. In the neurtonics calculation, the precursor drift solver implemented in PROTEUSNODAL was examined to demonstrate its capability to MSR applications.

This report is organized in the following manner: Section 2 provides a summary of multi-physics capability implemented using NEAMS tool for MSR application. Section 2 and 3 describes the demonstration of capability for the MCFR benchmark and 3D MSR test problem based on conceptual MSR core configuration, respectively. Conclusions are discussed in Section 4. 


\section{Summary of MSR Simulation Using NEAMS Tools}

\subsection{Coupling Scheme}

In the modeling and simulation for MSR using NEAMS tools, Nek5000 provides the accurate temperature and flow fields and PROTEUS-NODAL calculates the power/fission and precursor distribution. Because the velocity and temperature fields are also affected by the power distribution, the coupled calculation should be performed in the consistent manner. Therefore, the tight coupling is required by integrating two calculations with required data exchanges. Based on the fact that the power contribution from delayed precursor is very small, the practical calculation flow illustrated in Figure 2-1 was devised for coupling of Nek5000 and PROTEUS-NODAL. In this scheme, the initial PROTEUS-NODAL calculation is performed in the stationary fuel condition to generate the power distribution which is the input data of Nek5000 calculation. Then, the heat transfer and fluid dynamics calculations are performed using Nek5000 and the obtained temperature and velocity fields are provided back to the subsequent PROTEUS-NODAL calculation. The velocity field is used to compute the redistribution of precursor source. The temperature distribution would be used to update the cross section and material densities. In the current version of the coupling framework, the thermal feedback has not been implemented yet. Thus, only the velocity field is transferred to PROTEUS-NODAL. In the $2^{\text {nd }}$ PROTEUS-NODAL calculation, the flux and delayed precursor distribution are explicitly computed by performing the neutron diffusion and precursor drift solvers.

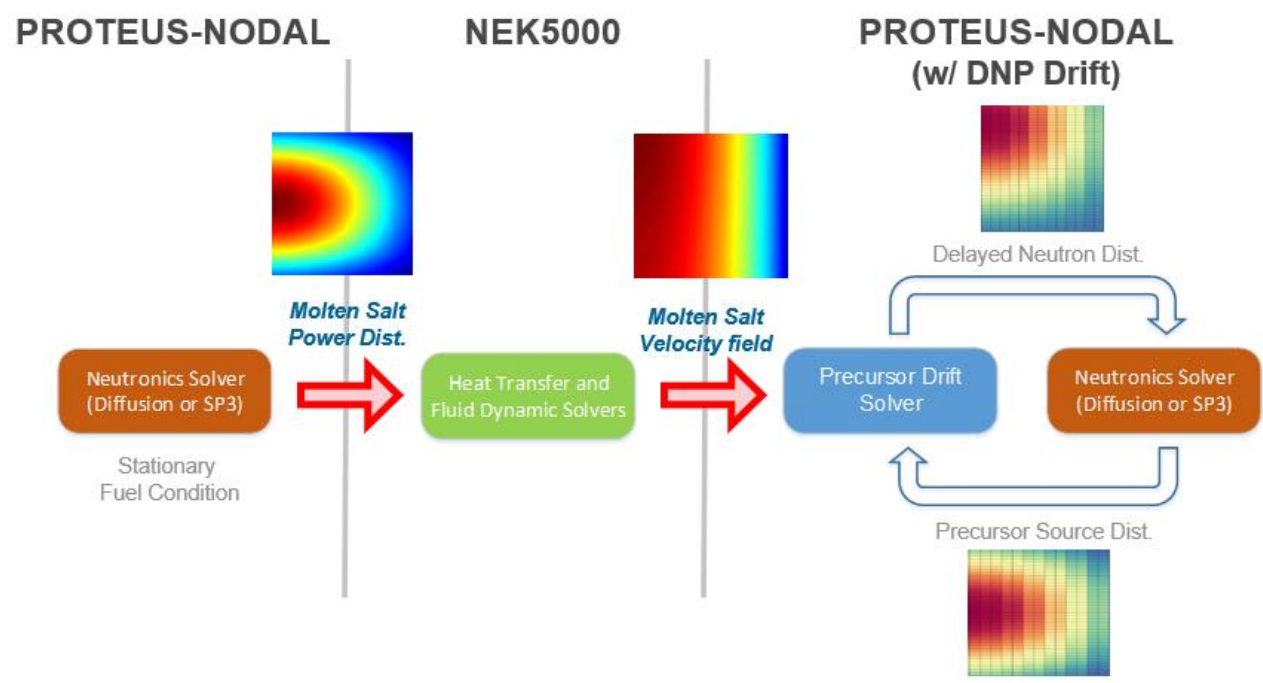

Figure 2-1. Calculation flow of Nek5000 and PROTEUS-NODAL coupled simulation [1].

\subsection{Implementation of Precursor Solver in PROTEUS}

The precursor drift solver solves the following governing equation:

$$
\nabla \cdot(\boldsymbol{u} C)+\lambda C=Q
$$

where $C, \lambda$ and $Q$ are the precursor concentration, decay constant and precursor source generation rate, respectively. $\boldsymbol{u}$ is the velocity field vector of flowing fuel. For brevity, the index for precursor family is omitted here. The precursor solver embedded into PROTEUS-NODAL solves Eq. (2.1) 
for structured and Cylindrical grid mesh by using finite difference scheme. For finite difference discretization, the following equation can be derived by integrating over a certain grid mesh:

$$
\sum_{j \in \partial V_{i}} \overline{(u \cdot n)_{i}^{j}} \bar{C}_{i}^{j} s_{i}^{j}+\lambda \bar{C}_{i} V_{i}=\bar{q}_{i} V_{i}
$$

where $i$ and $j$ are the indices for node and surface, respectively. $V$ is the volume of mesh, $\boldsymbol{n}$ is the normal vector of surface and $s$ is the surface area. The precursor concentration on the node surface $\bar{C}_{i}^{j}$ in Eq. (8) can be approximately expressed using the two adjacent node average concentrations. Alternatively, the node surface value can be expressed using the up-wind scheme based on the precursor flow direction on the corresponding surface. Optionally, the precursor diffusion can be included in the precursor drift model to incorporate the diffusion coefficients specified by the CFD results. By combining the discretized equations for each node and proper boundary conditions, the discretized system for precursor drift can be constructed and solved by using a linear system solver.

A numerical model for circulation of precursor through the external fuel loop was implemented in the precursor drift solver. In the neutronics calculation, the external fuel loop is not typical included in the computational domain due to the modeling complexity. Consequently, an appropriate treatment for boundary conditions that is imposed on the inlet and outlet surfaces is required to consider the precursor circulation. Under the assumption that the precursor concentration is uniform on the inlet surface, the inlet concentration can be computed for given the fixed circulation period and velocity field as:

$$
C_{\text {in }}\left(T_{r}\right)=\sum_{n=0}^{\infty}\left[\tilde{C}_{\text {out }} e^{-\lambda T_{r}}\left(P e^{-\lambda T_{r}}\right)^{n}\right]=\frac{\tilde{C}_{\text {out }} e^{-\lambda T_{r}}}{1-P e^{-\lambda T_{r}}}
$$

where $T_{r}$ is the circulation period, $\tilde{C}_{\text {out }}$ is the precursor concentration on the outlet surface computed with the zero precursor concentration on inlet surface, and $P$ is the probability that incoming precursor on inlet surface can leave the active core without decay. Using the precursor drift solver, $\tilde{C}_{\text {out }}$ and $P$ can be easily precomputed based on the solutions obtained by imposing the zero and unit precursor concentrations on the inlet boundary surface, respectively. Note that the circulation can be directly modeled without this additional treatment when the entire external loop is modelled in the calculation.

In the PROTEUS-NODAL calculation, a coarse mesh structure is typically used because the intranodal fluxes are described by high-order polynomials. In the precursor drift calculation, the refined mesh structure is required to correctly account for the crossflow effect. In the initial implementation, the same mesh structure was shared in the neutron diffusion and precursor drift calculation. The detailed modeling of precursor drift using the refined mesh structure might the significant computational burden on the nodal diffusion calculation, which made the overall calculation inefficient. As a remedy for this potential performance issue, the two-level mesh structure was introduced in the precursor drift calculations. In the two-level mesh structure, the refined mesh structure is used for precursor drift calculation to properly account for cross flow, while the neutron diffusion calculation is performed with the coarse mesh structure as illustrated in Figure 2-2. 

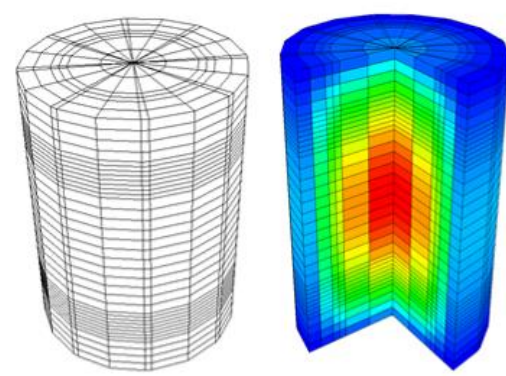

Coarse mesh structure for Neutron Diffusion
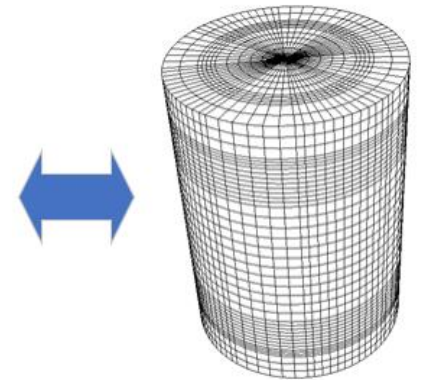

Refined mesh structure for Precursor Drift

Figure 2-2. Two-level mesh structure for PROTEUS-NODAL with precursor drift 


\section{MCFR Benchmark Problem}

To demonstrate the advanced multiphysics reactor modeling capabilities, Nek5000 and PROTEUSNODAL are utilized to address a MCFR test problem formulated by industry. In the problem, the physics includes predicting the re-distribution of delayed neutron precursors, and its influence on eigenvalue and effective delayed neutron fraction ( $\beta$-eff).

\subsection{Nek5000 Simulation}

A 2D core flow case is established as the starting point to test the coupling between the neutronics in PROTEUS-NODAL and the computational fluid dynamics (CFD) in Nek5000. This section will primarily focus on the investigations done in Nek5000. Figure 2-1 shows the computational mesh for the fluid region, and a reflector is modeled between the core and the outer channel corresponding to the blank region. The heat generation rate is assumed to be uniform inside the core $(x<1.0)$ according to the problem statement. The nominal power is removed at the upper channel marked by the yellow color as shown Figure 3-1. The symmetry boundary condition is applied to core centerline $(\mathrm{x}=0)$, while the rest of edges/boundaries are treated as no-slip walls. A momentum source is implemented at the upper part of the outer channel, which generates a pressure jump in the outer channel and drives the fuel flow to recirculate (as shown in Figure 3-2 and Figure 3-3).

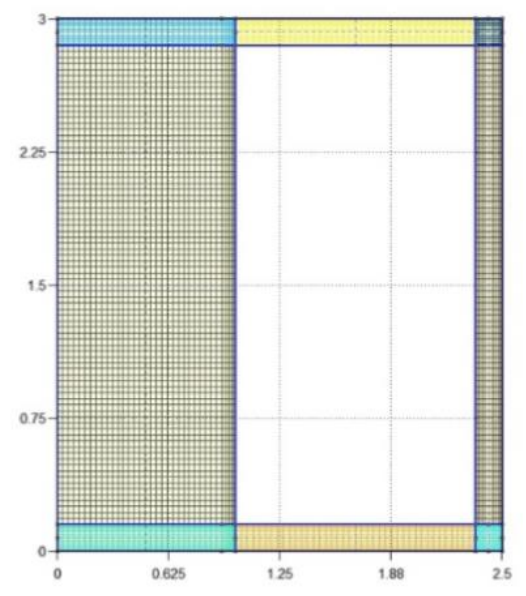

Figure 3-1. Computational mesh for 2D core flow case. 


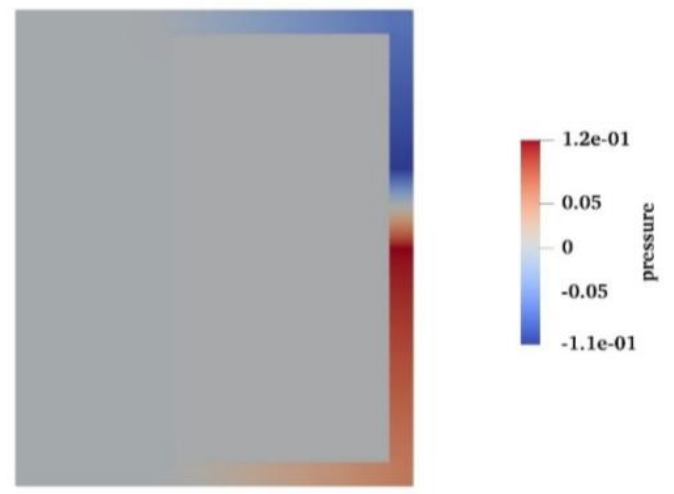

Figure 3-2. Momentum source generates a pressure jump in the outer channel which drives the flow recirculation.
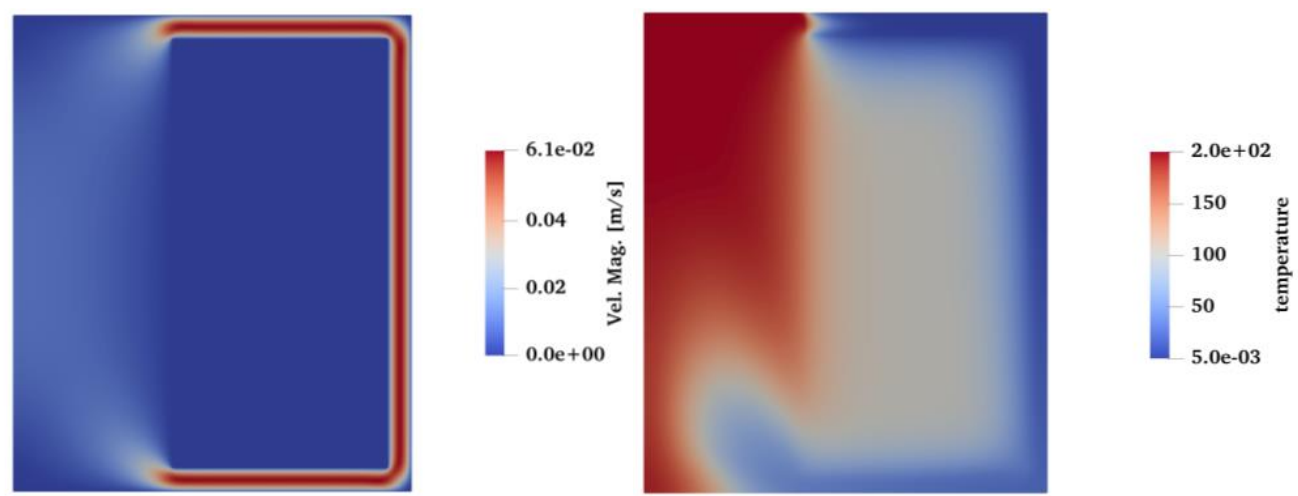

Figure 3-3. Velocity and temperature profiles in the laminar flow scenario.

A laminar flow scenario is first simulated with Nek5000. The mean vertical velocity in the core is $6.05 \mathrm{e}-3$, which leads to a Reynolds number of 6.05 . The steady state velocity and temperature profiles are illustrated in Figure 3-4. As expected, the temperature gradually builds up inside the core along the vertical direction. The fuel flow cools down as it passes through the upper channel and then returns to the core from the bottom channel. In addition to the temperature field, the Nek5000 can also solve the precursor concentrations from multiple precursor families for providing a reference solution to PROTEUS-NODAL. An accurate prediction of the precursor concentration field can be achieved by taking advantage of the precursor generation rates estimated by PROTEUS-NODAL. An auxiliary software is developed to readily convert the PROTEUSNODAL results into Nek5000 supported data format. Nek5000 can then load the needed data as input and perform the simulation. The precursor generation rates from PROTEUS-NODAL are exhibited in Figure 3-4, and all the precursor families have the same distribution profile but with various magnitude. The decay constants are listed in Table 1. A small decay constant indicates it is relatively slower for the corresponding precursor family to decay compared to the precursor family with larger decay constant. 


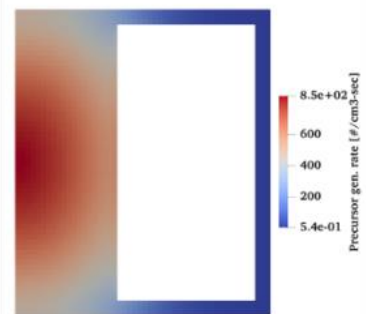

Precursor Family 01

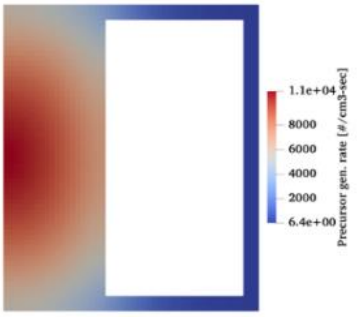

04

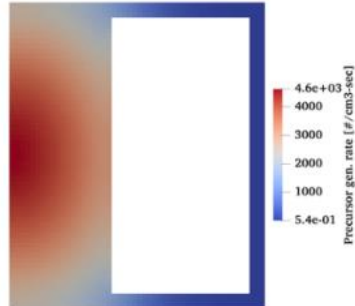

02

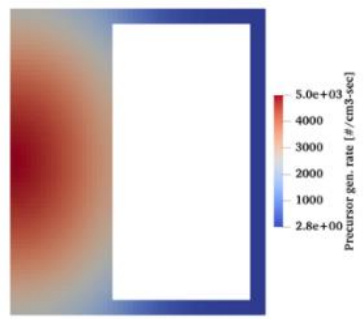

05

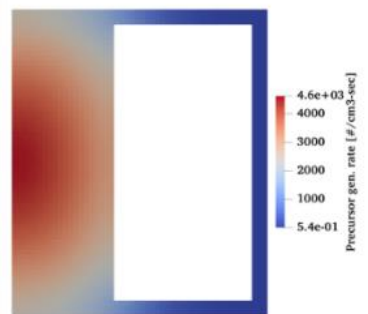

03

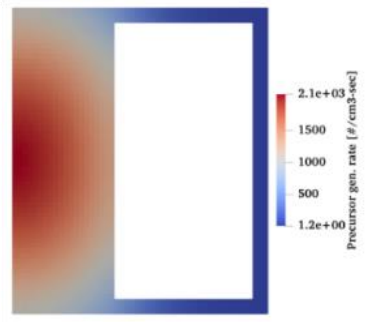

06

Figure 3-4. Precursor generation rates of different families inside the 2D core.

Table 1. Decay constant of precursors.

\begin{tabular}{cc}
\hline Precursor family & Decay constant $(\mathbf{1} / \mathbf{s})$ \\
\hline 1 & $1.34854 \mathrm{E}-02$ \\
2 & $3.18482 \mathrm{E}-02$ \\
3 & $1.22479 \mathrm{E}-01$ \\
4 & $3.17697 \mathrm{E}-01$ \\
5 & $8.94648 \mathrm{E}-01$ \\
6 & $3.00840 \mathrm{E}+00$ \\
\hline
\end{tabular}

Six additional scalar fields are specified in Nek5000 calculations that include all precursor families. The precursor generation rates and decay rates are incorporated in the source term, and a convection equation is solved for each precursor family based on the velocity field of the carrying fluid (i.e. the fuel fluid inside the 2D core). The precursor concentration gradually builds up till a dynamic equilibrium is established at steady state. Figure 3-5 illustrates the steady-state distributions of precursor concentration. In general, the advection effect plays a significant role in determining the overall precursor concentration profile. However, this effect would diminish when the precursor decays increasingly fast. As a result of the fast decay, the advection effect becomes negligible on the distribution of the precursor family 6 . Also, the precursors from most of the families decay away while traveling through the fluid channels (such as the precursor family 2 to 6 herein). On the other hand, with a much slower decay rate, the precursors from family 1 and 2 do not completely decay away and some remnants even return to the core after traveling the entire primary loop. This phenomenon could have some profound influence on the reactivity of which the further investigations may be needed. 


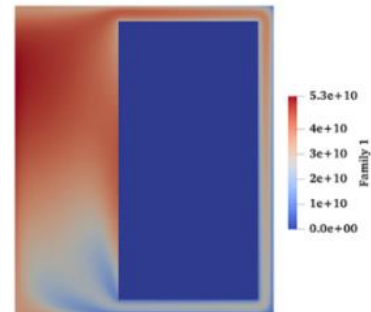

Precursor Family 01

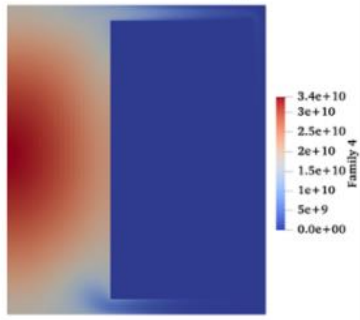

04

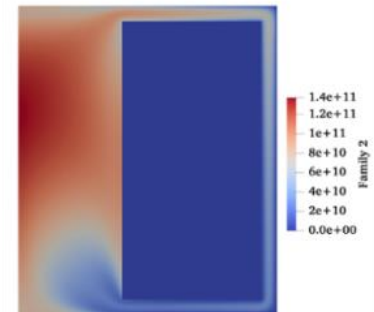

02

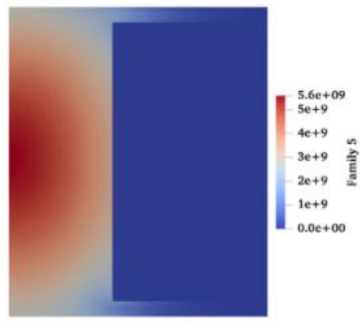

05

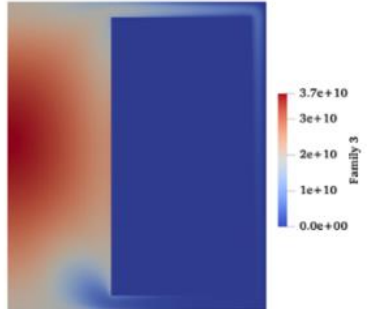

03

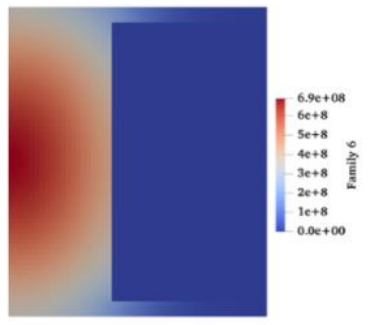

06

Figure 3-5. Steady-state precursor concentrations for different precursor families.

It has been demonstrated in the aforementioned laminar case study that Nek5000 is capable to solve the $2 \mathrm{D}$ core flow case with external input concerning the precursor generation rates. It lays a sound foundation for the subsequent studies focusing the prototypical reactor conditions. In the next step, realistic material properties are to be considered for both the fuel flow and the reflector. The fuel salt selected is $17 \% \mathrm{UCl}_{3}-71 \% \mathrm{UCl}_{4}-12 \% \mathrm{NaCl}_{\text {while }}$ the stainless-steel (SS) Type 316 is used for the reflector. The reference temperature is $900 \mathrm{~K}$. Key properties are listed in Table 2. Based on the material properties and the flow conditions identified in the problem statement, a series of important simulation parameters are evaluated as listed in Table 3, while will be employed in the corresponding Nek5000 calculations.

Table 2. Material properties of fuel salt and reflector at the reference temperature.

\begin{tabular}{ccc}
\hline Material Properties & Fuel Salt & Stainless Steel 316 \\
\hline Density $(\mathrm{kg} / \mathrm{m} 3)$ & 3682.76 & 8000 \\
Heat Capacity $(\mathrm{J} / \mathrm{kg}-\mathrm{K})$ & 474.816 & 500 \\
Thermal Conductivity $(\mathrm{W} / \mathrm{m}-\mathrm{K})$ & 1.46568 & 16.3 \\
Viscosity $\left(\mathrm{Pa}^{*}\right.$ s or kg/m-s) & 0.003698 & $\mathrm{n} / \mathrm{a}$ \\
\hline
\end{tabular}


Table 3. Simulation parameters corresponding to the prototypical core flow conditions.

\begin{tabular}{cc}
\hline Parameter & Value \\
\hline Reference Temperature $(\mathrm{K})$ & 900 \\
Core hydraulic diameter $(\mathrm{m})$ & 2 \\
Mean velocity in the core $(\mathrm{m} / \mathrm{s})$ & 0.73 \\
\hline Reynolds number & $1.46 \mathrm{E}+06$ \\
Prandtl number & 1.20 \\
Peclet number & $1.75 \mathrm{E}+06$ \\
rhoCp $(\mathrm{J} / \mathrm{m} 3-\mathrm{K})$ & $1.75 \mathrm{E}+06$ \\
Heat generation rate $(\mathrm{W} / \mathrm{m} 3)$ & $2.65 \mathrm{E}+07$ \\
\hline Non-dimensionalized heat generation rate & $4.59 \mathrm{E}-02$ \\
Dimensionless heat conductivity of SS & 11.12 \\
Dimensionless rhoCp of SS & 2.29 \\
\hline
\end{tabular}

\subsection{PROTEUS Simulation}

\section{D PROTEUS Model}

The 2D MCFR benchmark problem was prepared for testing the coupled simulation capability. The active core is configured with the homogeneous fuel region having $100 \mathrm{~cm}$ width and $300 \mathrm{~cm}$ height. The external pipe having $15 \mathrm{~cm}$ width is explicitly represented in the model. The symmetric boundary condition is applied to core center. Figure 3-6 shows the actual geometry model for PROTEUS-NODAL calculations. The properties of molten salt fuel used in this benchmark problem are summarized in Table 4. The 33-group cross section data and 6-family delayed neutron parameters were generated using $\mathrm{MC}^{2}-3$ [4]. The velocity fields were generated at multiple Reynold numbers to investigate the precursor movements at various flow conditions.

Table 4. Fuel Properties of MCFR benchmark problem.

\begin{tabular}{cc}
\hline Property & Value \\
\hline Salt composition & $17 \% \mathrm{UCl}_{3}-71 \% \mathrm{UCl}_{4}-12 \% \mathrm{NaCl}$ \\
Enrichment & $20 \mathrm{wt} \%$ \\
Temperature & $725 \mathrm{C}$ \\
Density & $3.461 \mathrm{~g} / \mathrm{cc}$ \\
\hline
\end{tabular}




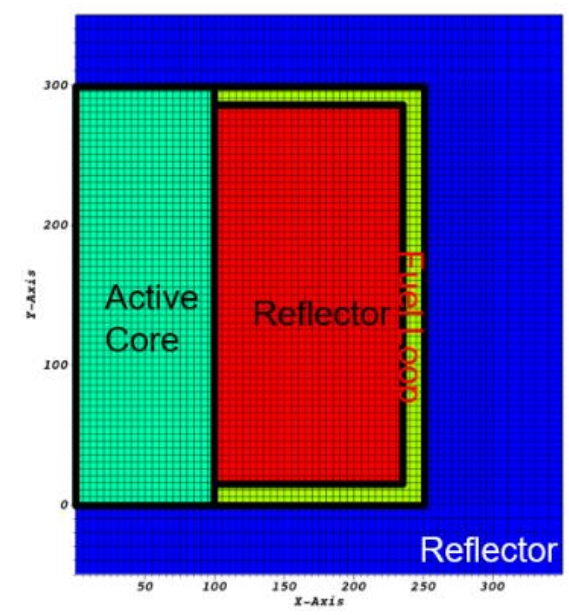

Figure 3-6. PROTEUS model for MCFR benchmark problem.

\section{Examinations of Precursor Drift}

To examine precursor drift solver implemented in PROTEUS-NODAL, the MCFR benchmark problem was solved for the several flow conditions computed by Nek5000, which are summarized in Table 5. Additionally, the stationary fuel case was also solved for comparing against the flowing cases. The obtained results are summarized in Table 6, Figure 3-7 and Figure 3-8. As shown in Table 6, the eigenvalues of moving fuel cases decreased compared with the stationary one because of neutron losses from precursor drift. The eigenvalues decreased as increasing the fuel flow velocities. When the flow rate increased from $0.01 \%$ to $10 \%$, the circulation time through the external loop is reduced and the recirculation zone of precursor become pronounced as shown in Figure 3-8, which resulted in eigenvalue increase around $10 \mathrm{pcm}$.

Table 5. Flow conditions for MCFR benchmark problem.

\begin{tabular}{|c|c|c|}
\hline Case & Re number & Remark \\
\hline Stationary & - & - \\
\hline Very low flow rate & 6.00 & Laminar flow \\
\hline $0.01 \%$ flow rate ${ }^{a)}$ & $1.42 \mathrm{E}+2$ & Turbulent flow \\
\hline $10 \%$ flow rate ${ }^{a)}$ & $1.42 \mathrm{E}+3$ & Turbulent flow \\
\hline
\end{tabular}

a) Re number of nominal flow: $1.42 \mathrm{E}+5$

Table 6. k-eff for MCFR benchmark problem.

\begin{tabular}{lll}
\hline Case & $\boldsymbol{k}$-eff & Dif. $\boldsymbol{k}$-eff, $\boldsymbol{p c m}$ \\
\hline Stationary & 1.18426 & - \\
Very low flow rate & 1.18405 & -21 \\
$0.01 \%$ flow rate & 1.18267 & -159 \\
$10 \%$ flow rate & 1.18279 & -147 \\
\hline
\end{tabular}




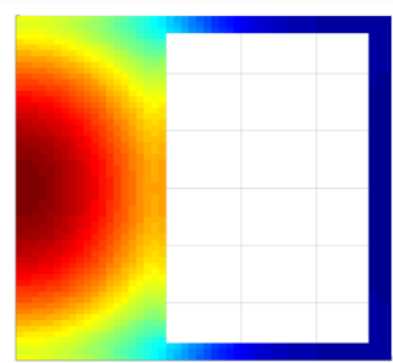

(a) Stationary

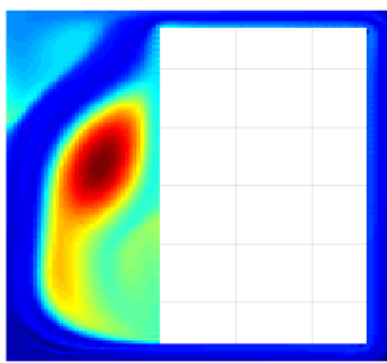

(c) $0.01 \%$ flow rate

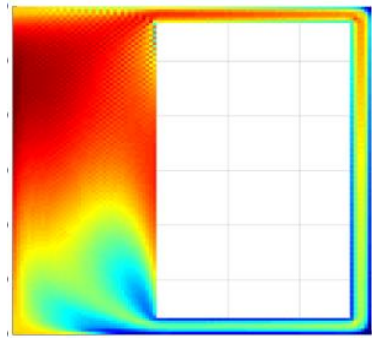

(b) Very low flow rate

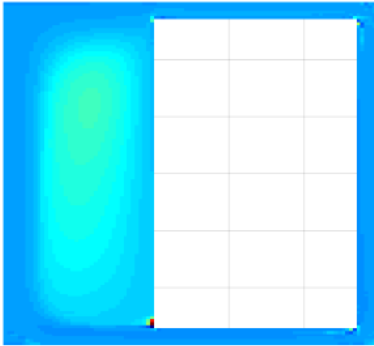

(d) $10 \%$ flow rate

Figure 3-7. Precursor distribution of family 1 for MCFR benchmark problem.

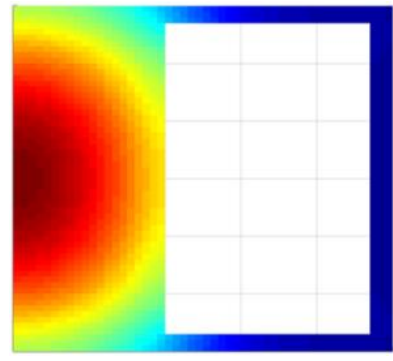

(a) Stationary

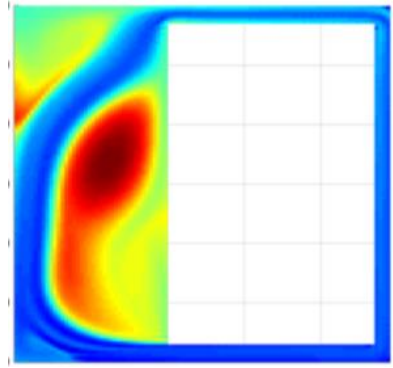

(c) $0.01 \%$ flow rate

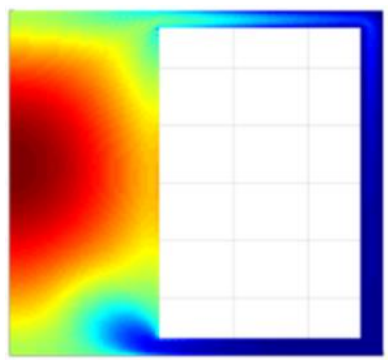

(b) Very low flow rate

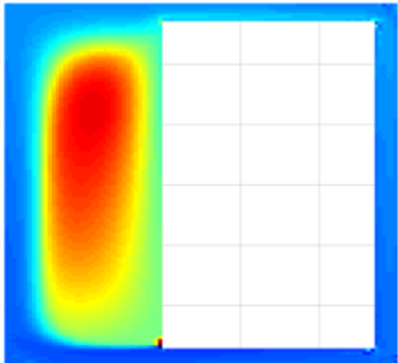

(d) $10 \%$ flow rate

Figure 3-8. Precursor distribution of family 3 for MCFR benchmark problem.

To verify the precursor solver of PROTEUS-NODAL, the reference precursor distributions were obtained using Nek5000 for the case with the very low flow rate. In this Nek5000 calculation, the precursor source distribution of PROTEUS-NODAL was provided along with the precursor decay constants for consistent comparisons. The obtained results of Nek5000 calculations are summarized in Figure 3-9, and they matched well with the reference precursor distribution from Nek5000. 


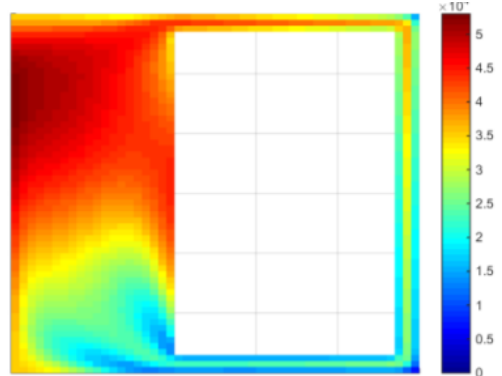

(a) Nek5000 Family 1

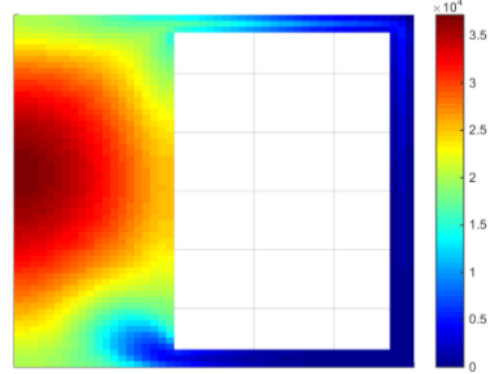

(c) Nek5000 Family 3

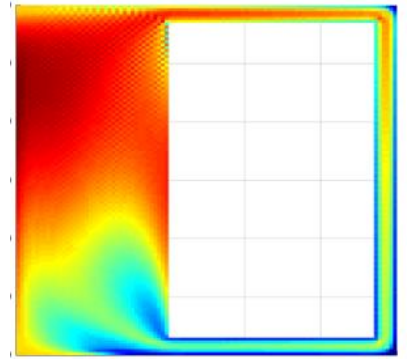

(b) NODAL Family 1

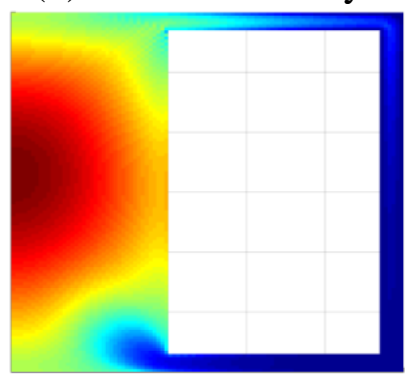

(d) NODAL Family 3

Figure 3-9. Comparisons of precursor distributions obtained using Nek5000 and PROTEUSNODAL Solution.

\section{$\underline{\text { Reactivity Parameters }}$}

As demonstrating the precursor drift solver for MSR application, the reactivity parameters were computed using PROTEUS-NODAL. Prior to compute the parameters on the flowing fuel conditions, the various parameters were computed with stationary fuel conditions as summarized in Table 7. Then, the effective delayed neutron fraction ( $\beta$-eff) was calculated with the three flowing conditions that were used for the tests of precursor drift. As shown in Table 8, the obtained $\beta$-eff values are reduced as increasing the fuel flow rate, which is consistent with the eigenvalue trend.

Table 7. Reactivity parameters of MCFR benchmark problem with stationary fuel condition.

\begin{tabular}{ll}
\hline Parameter & Value \\
\hline k-eff & 1.18426 \\
$\beta$-eff & $779 \mathrm{pcm}$ \\
Doppler Coefficient & $1.01 \mathrm{pcm} / \mathrm{K}$ \\
Fuel Density Coefficient & $13.99 \mathrm{pcm} / \mathrm{K}$ \\
\hline
\end{tabular}

Table 8. $\beta$-eff of MCFR benchmark problem wits flowing fuel conditions.

\begin{tabular}{llll}
\hline Case & Re number & $\beta$-eff, pcm & k-eff, pcm \\
\hline Stationary & - & 779 & 1.18426 \\
Very low flow rate & 6.00 & 761 & 1.18405 \\
$0.01 \%$ flow rate & $1.42 \mathrm{E}+2$ & 663 & 1.18267 \\
$10 \%$ flow rate & $1.42 \mathrm{E}+3$ & 672 & 1.18279 \\
\hline
\end{tabular}




\section{3D MSR Test Problem with 4 Loops}

A test problem was designed to extends the coupled simulation capabilities to realistic 3D MSR problem. A cylindrical core configuration was selected because it is the most-common design of fast-spectrum MSR and the inlet and outlet pipes are explicitly represented in the model. By performing this 3D MSR test problem, the coupled simulation capability could be demonstrated with detailed precursor drift modeling.

\subsection{Nek5000 Simulation}

Figure 4-1 shows the computational domain where the flow enters the core from four bottom pipes and exits through the top ones. The ratio of core diameter over inlet/outlet pipe diameter is 4:1. With the focus on obtaining accurate flow field data inside the core, the CFD simulations do not account for external loop components yet, such as the heat exchangers and pumps. It is possible to add these components later in CFD investigations through reduced-order models, such as the porous media model for heat exchanger. Although the inlet pipes have been arranged to avoid head-on collision of opposite jets, the current domain is not particularly optimized to minimize the flow recirculation.

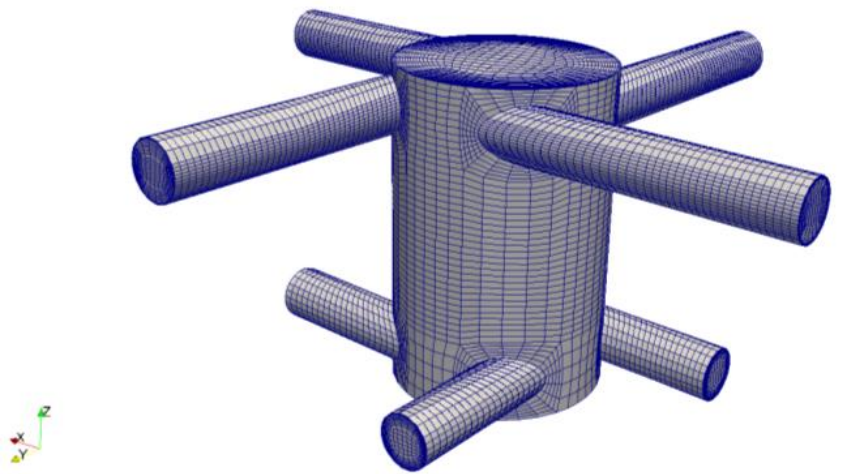

Figure 4-1. Computational mesh for a 3D MSR core with inlet (bottom) and outlet (top) pipes.

The CFD simulations of core flow are conducted at two different Reynolds numbers to cover both the laminar regime $(\operatorname{Re}=125)$ and turbulent regime $(\operatorname{Re}=3.65 \mathrm{e}+4)$. The direct numerical simulation (DNS) is performed for the laminar case while an $\kappa-\tau$ Reynolds-averaged Navier Stokes (RANS) model is employed for the turbulent case. An analytical precursor source has been implemented in Nek5000 with a decay rate set to 3.2036e-2. The velocity field of the laminar case is shown in Figure 4-2. It is noticed that the velocity magnitude is much smaller in the core center. A closer look at the vertical velocity (Figure 4-3) shows that the flow moves upward in the near wall region while slightly moving downward in the central region. As one can see in Figure 4-4, the downward flow movement in the core center has a direct impact on the precursor profile. As a result, the precursor peak is shifted downward accordingly. 


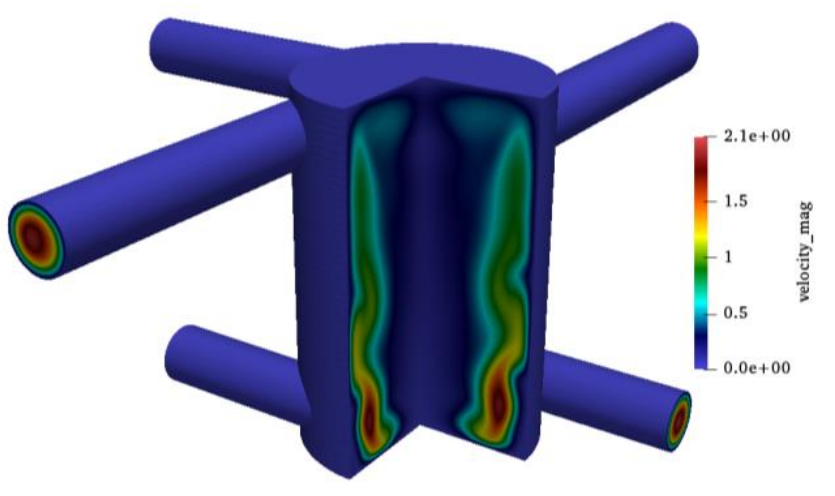

Figure 4-2. Velocity field inside the core for $\operatorname{Re}=125$.

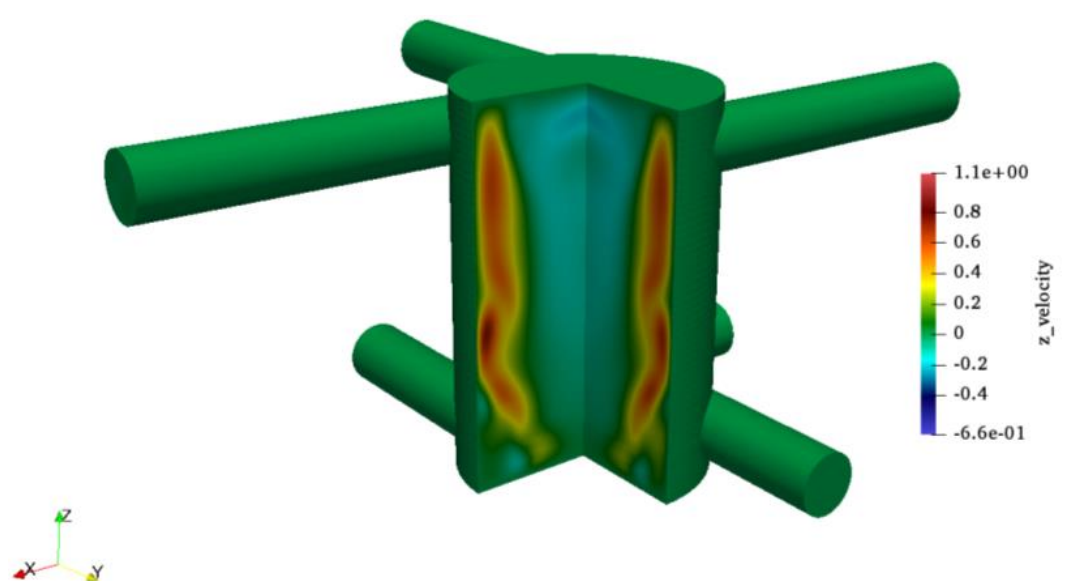

Figure 4-3. Vertical velocity inside the core for $\mathrm{Re}=125$.

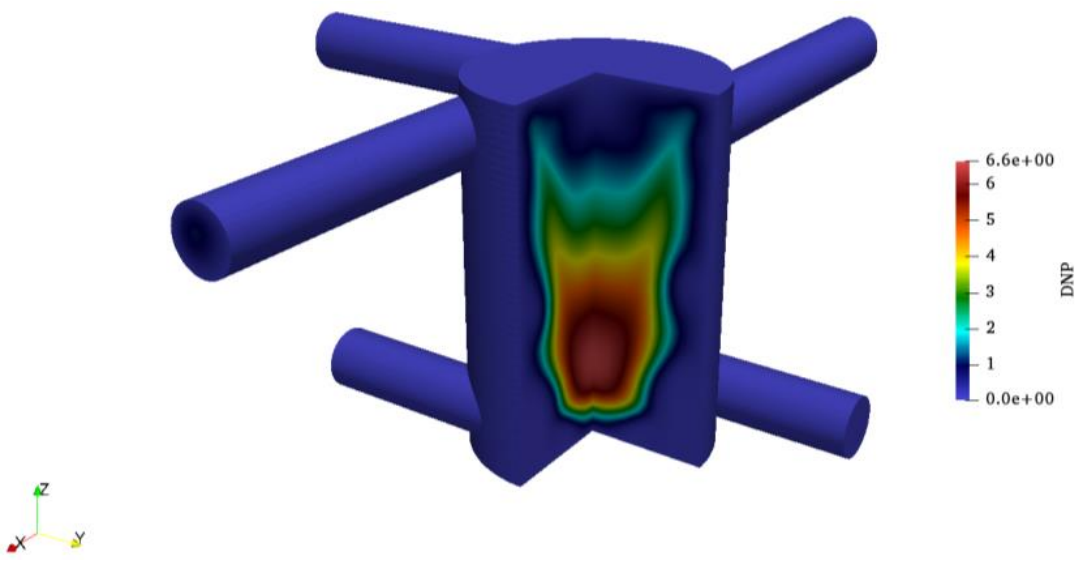

Figure 4-4. Precursor field inside the core for $\operatorname{Re}=125$.

The flow field and precursor solutions for the turbulent case are shown from Figure 4-5 to Figure 4-6. As the Reynolds number goes up, the velocity field becomes quite fluctuating and strong vortices are formed as the flow enters the core. The core geometry optimization is required to 
minimize large-scale recirculations that could induce temporal or spatial instabilities in reactor power distribution.

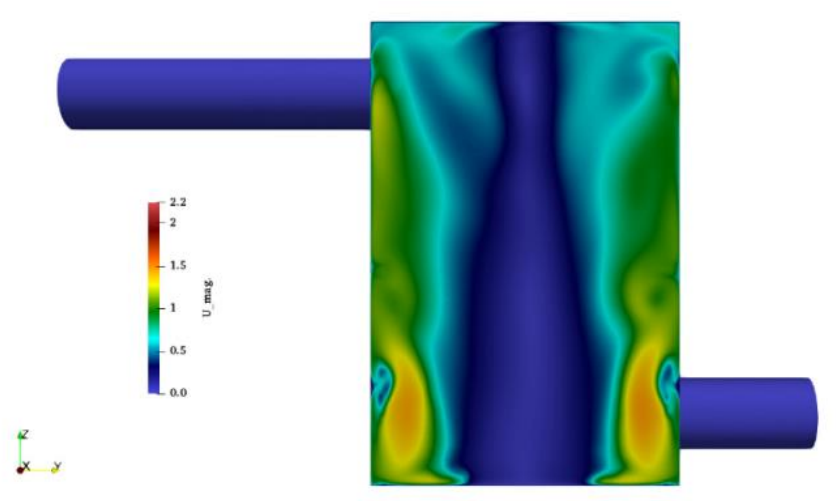

Figure 4-5. Velocity field inside the core for $\mathrm{Re}=3.65 \mathrm{e}+4$.

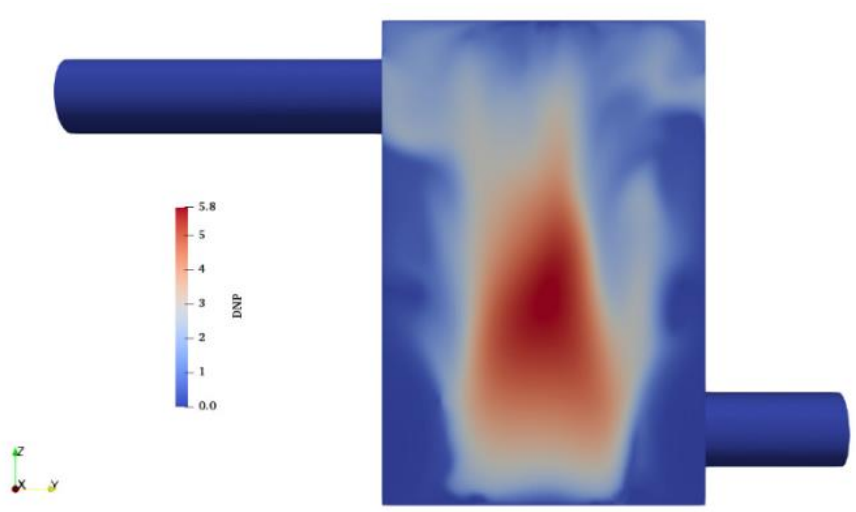

Figure 4-6. Precursor field inside the core for $\mathrm{Re}=3.65 \mathrm{e}+4$.

\subsection{PROTEUS Simulation}

\section{PROTEUS Models}

In this 3D MSR test problem, the active core is cylindrical shape having $1.0 \mathrm{~m}$ diameter and $1.5 \mathrm{~m}$ height and the four inlet and outlet pipes with $0.25 \mathrm{~m}$ diameter are attached top and bottom of active core. Figure 4-7 shows the modeling of 3D MSR test problem for PROTEUS-NODAL calculation. The active core region is explicitly represented using the $\mathrm{R}-\theta-\mathrm{Z}$ geometry option and the reflector regions were added to the model for realistic neutronics calculation. The mesh structure for PROTEUS-NODAL calculation is shown in R- $\theta-Z$. The inlet and outlet legs are not included due to the modeling limitation of PROTEUS-NODAL and the intersection of these pipes on the active core outer boundaries are approximately represented using the R- $\theta-Z$ meshes as shown in Figure 4-7. The decay of precursor in the external loops is implicitly modeled using the precursor circulation option of drift solver. 

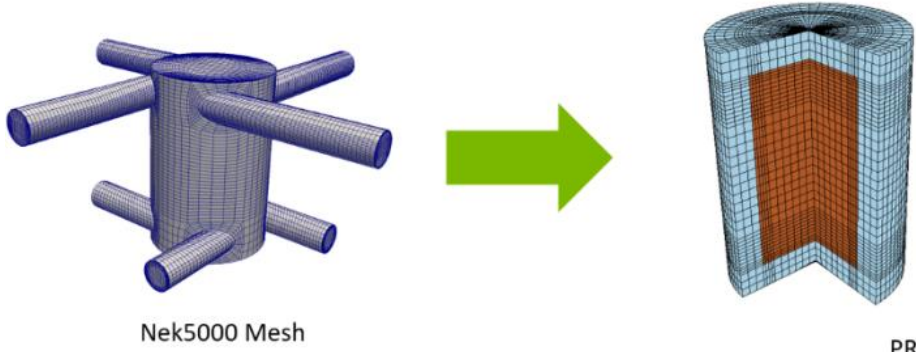

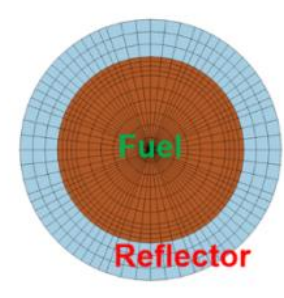

PROTEUS Mesh

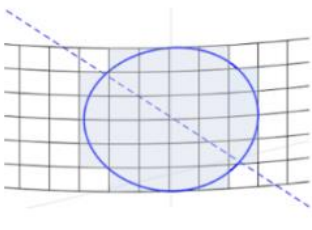

Inlet and Outlet Modeling in PROTEUS

Figure 4-7. Modeling of PROTEUS-NODAL for 3D MSR test problem with 4 loops.

\section{Precursor Drift Model Tests}

The PROTEUS-NODAL calculations were focused on demonstrating the precursor drift modeling capability on the realistic core configuration. For flowing fuel simulation, the composition and properties of fuel salt given in Table 4 was used. The Nek5000 generated velocity field with Reynold Number of 125.0 and the precursor source distribution yielded from Nek5000 and NODAL diffusion calculations are transferred to the mesh grid of precursor calculation as shown in Figure 4-8. Since the external fuel loop was excluded in the PROTEUS-NODAL, the precursor movement in the external piper was also considered by imposing additional boundary conditions on the precursor solver. In the PROTEUS-NODAL calculation, the precursor solution was obtained using the fine mesh structure and the neutron diffusion calculation was done using the relatively coarse mesh structure as illustrated in Figure 2-2. Note the nodal calculation does not required to use the fine mesh structure due to the high-order representation of intra-nodal solution. The 33group cross section data and 6-family of delayed neutron parameters were used for both neutron diffusion and precursor drift calculations.

The base calculation of PROTEUS-NODAL was performed with the $10 \mathrm{sec}$ of precursor circulation period, which means that $10 \mathrm{sec}$ is taken to the fuel salt for returning to the active core region through passing the external pipe regions. The additional calculations were performed with the different circulation periods to investigate its impact on the precursor distribution and eigenvalues. The obtained results of PROTEUS-NODAL calculations were summarized in Figure 4-9 and Figure 4-10 and Table 9. In the base calculation results shown in Figure 4-9, the core center has significantly higher precursor concentration than the periphery regions due to the recirculation zone of flowing fuels. Note that the thermal feedback was not considered in the neutronics and fluid calculations. If the thermal feedback is properly incorporated in the calculation, the fuel flow will be enhanced toward to periphery. The precursor source generation rate in the core center will be reduced as well due to temperature increase and fuel density decrease. Consequently, it is expected that the higher precursor distribution in the core center would be less pronounced. Figure 4-10 shows the changes of precursor distribution with respect to the different circulation period: 1, 10, 50 and $100 \mathrm{sec}$. As increasing the fuel residence time in the external pipe region, the precursor concentration in the periphery region is reduced. However, the precursor concentration in the core center region is solely determined from the fuel recirculation pattern which is not affected by the circulation period. Thus, the precursor concentration in the core center region remain almost same with change of circulation period as shown in Figure 4-10. The eigenvalue changes are summarized in Table 9. As expected,

the loss of reactivity and subsequent reduction in the $\beta$-eff are more pronounced with a longer circulation period. This reduction can significantly impact the ability to control the reactor during 
transient and accident scenarios if additional design measures for control are not taken. Note that this $\beta$-eff reduction is not that significant from 10 to 50 seconds, which can be very informative for the ex-core loop design. This can be taken into account along with the recommendation that MSRs be designed to minimize the ex-core volume to reduce the fuel salt required to start up.

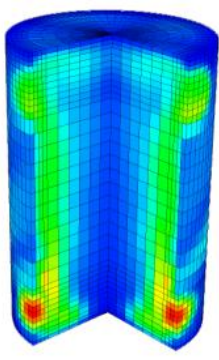

Fuel Velocity

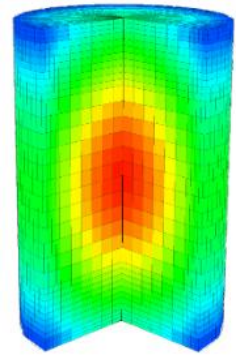

Precursor Source Distribution

Figure 4-8. Velocity and precursor distributions used in precursor drift calculations for 3D MSR test problem.

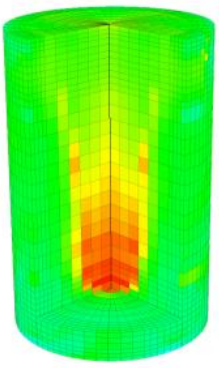

Family 2

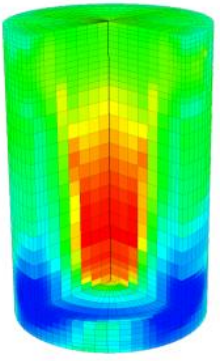

Family 4

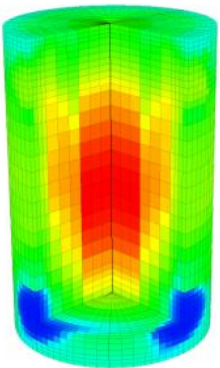

Family 6

Figure 4-9. Precursor distribution of 3D MSR test problem (circulation period of $10 \mathrm{sec}$ ).

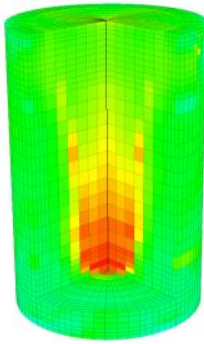

$10 \mathrm{sec}$

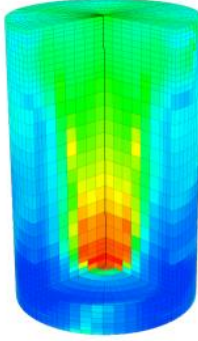

$50 \mathrm{sec}$

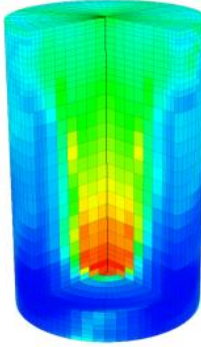

$100 \mathrm{sec}$

Figure 4-10. Changes of precursor distributions of 3D MSR test problem with Different circulation period (Family 2). 
Table 9. Eigenvalue results of 3D MSR test problem.

\begin{tabular}{ccccc}
\hline Case & Circulation Period, sec & Eigenvalue & Dif. $\boldsymbol{k}$-eff, pcm & $\boldsymbol{\beta}$-eff, pcm \\
\hline Stationary & - & 0.89874 & - & 753 \\
\hline \multirow{4}{*}{ Flowing } & 1 & 0.89789 & 85 & 660 \\
& 10 & 0.89640 & 234 & 468 \\
& 50 & 0.89603 & 271 & 421 \\
& 100 & 0.89600 & 274 & 417 \\
\hline
\end{tabular}




\section{Conclusions}

In this study, a coupled simulation capability of Nek5000 and PROTEUS-NODAL was successfully employed to simulate the two conceptual MSR core problems to demonstrate that the NEAMS tool can be applicable for modeling and simulation of MSR applications. In the coupled simulation, Nek5000 and PROTEUS-NODAL provided the accurate and detailed flow field and power/flux distributions along with the delayed neutron precursor distributions.

The coupled simulations as effort to demonstrate the capabilities were focused on predicting the non-uniform distribution of delayed neutron precursor and investigating the influence of on eigenvalues and other reactivity parameters. For the MCFR benchmark problem, the Nek5000 and PROTEUS-NODAL calculations were performed for the various flow conditions ranging from laminar to turbulent cases. The precursor drift model implemented in PROTEUS-NODAL was also verified by comparing against the reference precursor distribution generated from Nek5000. Along with the MCFR benchmark problem, the 3D test problem based on the conceptual MSR design was solved to confirm the coupled simulation capability in the realistic MSR core configurations. The coupled simulation tests demonstrated this approach's capabilities to identify recirculation zones and quantify the reduction in the effective delayed neutron fraction, both of which are very important design considerations for future fast MSRs. It was also suggested the thermal feedback should be incorporated in the simulations to correctly modeling the flow fields and precursor drift by accounting for complicated fuel flowing patterns such as recirculation zone. 


\section{References}

1. B. FENG, T. FEI, D. SHAVER, Y.S. JUNG, J. FANG, R. RAHAMAN, C.H. LEE, M.A. SMITH, and E.R. SHEMON, "Multiphysics Modeling of Precursors in Molten Salt Fast Reactors using PROTEUS and Nek5000," Proc. PHYSOR 2020, Cambridge, UK, Mar. 30-Apr. 2 (2020).

2. Nek5000 Version 17.0. Dec. 17, 2017. Argonne National Laboratory, Illinois. Available: https://nek5000.mcs.anl.gov.

3. Y.S. JUNG, M. A. SMITH and C. H. Lee, "PROTEUS-NODAL User Manual," ANL/NE-18/4, Argonne National Laboratory, February, 2018.

4. C. H. LEE, Y. S. JUNG, and W. S. YANG, "MC²-3: Multigroup Cross Section Generation Code for Fast Reactor Analysis," ANL/NE-11/41 (Rev.3), Argonne National Laboratory, August, 2018. 
Argonne

Nuclear Science and Engineering Division

Argonne National Laboratory

9700 South Cass Avenue, Bldg. 208

Argonne, IL 60439

www.anl.gov

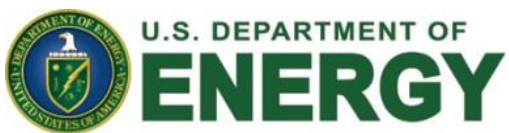

Argonne National Laboratory is a U.S. Department of Energy

laboratory managed by UChicago Argonne, LLC 